\title{
UV-induced oxo $\rightarrow$ hydroxy unimolecular proton-transfer reactions in hypoxanthine
}

Anna Gerega, Leszek Lapinski, Maciej J. Nowak and Hanna Rostkowska

\section{Supporting Information}


Chart S1. Atom numbering for the oxo-N(9)-H, oxo-N(7)-H, hydroxy-N(9)-H and hydroxy-N(7)-H tautomers of hypoxanthine.
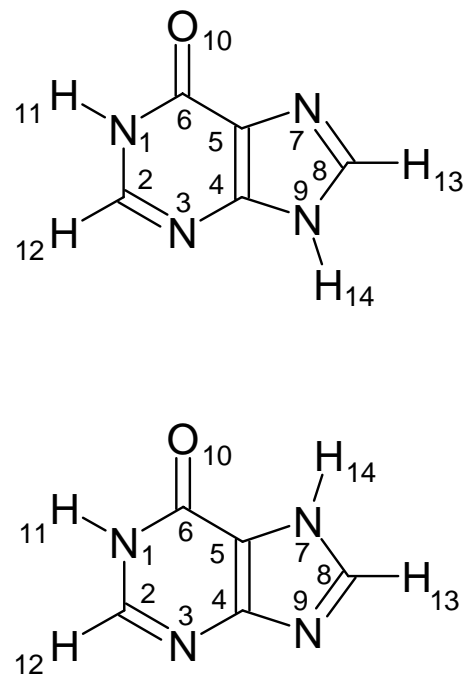

II<smiles></smiles>

III<smiles>Cc1nc(O[14CH3])c2c(n1)nc(C)n2C</smiles> 
Table S1. Internal coordinates used in the normal mode analysis for hypoxanthine forms I and II (atom numbering as in Chart S1)

\begin{tabular}{|c|c|c|}
\hline \multicolumn{3}{|l|}{ In-plane } \\
\hline \multicolumn{3}{|l|}{ Ring stretchings } \\
\hline $\mathrm{S}_{1}=\mathrm{r}_{1,2}$ & & $v \mathrm{~N} 1 \mathrm{C} 2$ \\
\hline $\mathrm{S}_{2}=\mathrm{r}_{2,3}$ & & $v \mathrm{C} 2 \mathrm{~N} 3$ \\
\hline $\mathrm{S}_{3}=\mathrm{r}_{3,4}$ & & $v \mathrm{~N} 3 \mathrm{C} 4$ \\
\hline $\mathrm{S}_{4}=\mathrm{r}_{4,5}$ & & $v \mathrm{C} 4 \mathrm{C} 5$ \\
\hline $\mathrm{S}_{5}=\mathrm{r}_{5,6}$ & & $v \mathrm{C} 5 \mathrm{C} 6$ \\
\hline $\mathrm{S}_{6}=\mathrm{r}_{6,1}$ & & $v \mathrm{C} 6 \mathrm{~N} 1$ \\
\hline $\mathrm{S}_{7}=\mathrm{r}_{5,7}$ & & $v \mathrm{C} 5 \mathrm{~N} 7$ \\
\hline $\mathrm{S}_{8}=\mathrm{r}_{7,8}$ & & $v \mathrm{~N} 7 \mathrm{C} 8$ \\
\hline $\mathrm{S}_{9}=\mathrm{r}_{8,9}$ & & $v \mathrm{C} 8 \mathrm{~N} 9$ \\
\hline $\mathrm{S}_{10}=\mathrm{r}_{9,4}$ & & $v \mathrm{~N} 9 \mathrm{C} 4$ \\
\hline \multicolumn{3}{|l|}{ Stretching CO } \\
\hline $\mathrm{S}_{11}=\mathrm{r}_{10,6}$ & & $v \mathrm{C}=\mathrm{O}$ \\
\hline \multicolumn{3}{|l|}{ Stretching $\mathrm{CH}, \mathrm{NH}$} \\
\hline $\mathrm{S}_{12}=\mathrm{r}_{11,1}$ & & $v \mathrm{~N} 1 \mathrm{H}$ \\
\hline $\mathrm{S}_{13}=\mathrm{r}_{12,2}$ & & $v \mathrm{C} 2 \mathrm{H}$ \\
\hline $\mathrm{S}_{14}=\mathrm{r}_{13,8}$ & & $v \mathrm{C} 8 \mathrm{H}$ \\
\hline $\mathrm{S}_{15}=\mathrm{r}_{14,9}$ & I & $v \mathrm{~N} 9 \mathrm{H}$ \\
\hline $\mathrm{S}_{15}^{\prime}=\mathrm{r}_{14,7}$ & II & $v \mathrm{~N} 7 \mathrm{H}$ \\
\hline \multicolumn{3}{|l|}{ Ring in-plane deformation } \\
\hline$S_{16}=\left(6^{-1 / 2}\right)\left(\beta_{1,3,2}-\beta_{2,4,3}+\beta_{3,5,4}-\beta_{4,6,5}+\beta_{5,1,6}-\beta_{6,2,1}\right)$ & & $\beta \mathrm{R} 1$ \\
\hline$S_{17}=\left(12^{-1 / 2}\right)\left(2 \beta_{1,3,2}-\beta_{2,4,3}-\beta_{3,5,4}+2 \beta_{4,6,5}-\beta_{5,1,6}-\beta_{6,2,1}\right)$ & & $\beta \mathrm{R} 2$ \\
\hline$S_{18}=(1 / 2)\left(\beta_{2,4,3}-\beta_{3,5,4}+\beta_{5,1,6}-\beta_{6,2,1}\right)$ & & $\beta \mathrm{R} 3$ \\
\hline $\mathrm{S}_{19}=\left(\left(1+2 a^{2}+2 b^{2}\right)^{-1 / 2}\right)\left(\beta_{7,9,8}+a\left(\beta_{5,8,7}+\beta_{8,4,9}\right)+b\left(\beta_{4,7,5}+\beta_{9,5,4}\right)\right)$ & & $\beta \mathrm{R} 4$ \\
\hline $\mathrm{S}_{20}=\left(\left(2(a-b)^{2}+2(1-a)^{2}\right)^{-1 / 2}\right)\left((a-b)\left(\beta_{5,8,7}-\beta_{8,4,9}\right)+(1-a)\left(\beta_{4,7,5}-\beta_{9,5,4}\right)\right)$ & & $\beta \mathrm{R} 5$ \\
\hline \multicolumn{3}{|l|}{ Bendings $\mathbf{C O}, \mathbf{C H}, \mathbf{N H}$} \\
\hline$S_{21}=\left(2^{-1 / 2}\right)\left(\beta_{1,10,6}-\beta_{5,10,6}\right)$ & & $\beta \mathrm{C}=\mathrm{O}$ \\
\hline$S_{22}=\left(2^{-1 / 2}\right)\left(\beta_{2,11,1}-\beta_{6,11,1}\right)$ & & $\beta \mathrm{N} 1 \mathrm{H}$ \\
\hline $\mathrm{S}_{23}=\left(2^{-1 / 2}\right)\left(\beta_{3,12,2}-\beta_{1,12,2}\right)$ & & $\beta \mathrm{C} 2 \mathrm{H}$ \\
\hline$S_{24}=\left(2^{-1 / 2}\right)\left(\beta_{7,13,8}-\beta_{9,13,8}\right)$ & & $\beta \mathrm{C} 8 \mathrm{H}$ \\
\hline $\mathrm{S}_{25}=\left(2^{-1 / 2}\right)\left(\beta_{8,14,9}-\beta_{4,14,9}\right)$ & $\mathbf{I}$ & $\beta \mathrm{N} 9 \mathrm{H}$ \\
\hline $\mathrm{S}_{25}^{\prime}=\left(2^{-1 / 2}\right)\left(\beta_{5,14,7}-\beta_{8,14,7}\right)$ & II & $\beta \mathrm{N} 7 \mathrm{H}$ \\
\hline \multicolumn{3}{|l|}{ Out-of-plane } \\
\hline \multicolumn{3}{|l|}{ Wagging $\mathrm{CO}, \mathrm{NH}, \mathrm{CH}$} \\
\hline $\mathrm{S}_{26}=\gamma_{10,5,6,1}$ & & $\gamma \mathrm{C}=\mathrm{O}$ \\
\hline $\mathrm{S}_{27}=\gamma_{11,6,1,2}$ & & $\gamma \mathrm{N} 1 \mathrm{H}$ \\
\hline $\mathrm{S}_{28}=\gamma_{12,1,2,3}$ & & $\gamma \mathrm{C} 2 \mathrm{H}$ \\
\hline $\mathrm{S}_{29}=\gamma_{13,7,8,9}$ & & $\gamma \mathrm{C} 8 \mathrm{H}$ \\
\hline $\mathrm{S}_{30}=\gamma_{14,8,9,4}$ & I & $\gamma \mathrm{N9H}$ \\
\hline $\mathrm{S}_{30}^{\prime}=\gamma_{14,5,7,8}$ & II & $\gamma \mathrm{N} 7 \mathrm{H}$ \\
\hline \multicolumn{3}{|l|}{ Ring torsions } \\
\hline$S_{31}=\left(6^{-1 / 2}\right)\left(\tau_{1,2,3,4}-\tau_{2,3,4,5}+\tau_{3,4,5,6}-\tau_{4,5,6,1}+\tau_{5,6,1,2}-\tau_{6,1,2,3}\right)$ & & $\tau \mathrm{R} 1$ \\
\hline$S_{32}=\left(12^{-1 / 2}\right)\left(2 \tau_{1,2,3,4}-\tau_{2,3,4,5}-\tau_{3,4,5,6}+2 \tau_{4,5,6,1}-\tau_{5,6,1,2}-\tau_{6,1,2,3}\right)$ & & $\tau \mathrm{R} 2$ \\
\hline$S_{33}=(1 / 2)\left(\tau_{2,3,4,5}-\tau_{3,4,5,6}+\tau_{5,6,1,2}-\tau_{6,1,2,3}\right)$ & & $\tau \mathrm{R} 3$ \\
\hline $\mathrm{S}_{34}=\left(\left(1+2 a^{2}+2 b^{2}\right)^{-1 / 2}\right)\left(\tau_{4,5,7,8}+b\left(\tau_{7,8,9,4}+\tau_{8,9,4,5}\right)+a\left(\tau_{5,7,8,9}+\tau_{9,4,5,7}\right)\right)$ & & $\tau \mathrm{R} 4$ \\
\hline $\begin{array}{l}\mathrm{S}_{35}=\left(\left(2(a-b)^{2}+2(1-a)^{2}\right)^{-1 / 2}\right)\left((a-b)\left(\tau_{9,4,5,7}-\tau_{5,7,8,9}\right)+(1-a)\left(\tau_{8,9,4,5}-\tau_{7,8,9,4}\right)\right) \\
\text { Two rings relative torsion }\end{array}$ & & $\tau$ R5 \\
\hline$S_{36}=\left(2^{-1 / 2}\right)\left(\tau_{6,5,49}-\tau_{7,5,4,3}\right)$ & & $\tau \mathrm{RR}$ \\
\hline
\end{tabular}

$r_{i, j}$ is the distance between atoms $A_{i}$ and $A_{j} ; \beta_{i, j, k}$ is the angle between vectors $A_{k} A_{i}$ and $A_{k} A_{j}$; $\tau_{i, j, k, 1}$ is the dihedral angle between the plane defined by $A_{i}, A_{j}, A_{k}$ and the plane defined by $A_{j}, A_{k}, A_{l}$ atoms; $\gamma_{i, j, k, l}$ is the angle between the vector $A_{k} A_{i}$ and the plane defined by atoms $A_{j}, A_{k}, A_{l}$.

$a=\cos 144^{\circ}=-0.8090 ; b=\cos 72^{\circ}=0.3090$ 
Table S2. Internal coordinates used in the normal mode analysis for hypoxanthine forms III and IV (atom numbering as in Chart S1)

\begin{tabular}{|c|c|c|}
\hline \multicolumn{3}{|l|}{ In-plane } \\
\hline \multicolumn{3}{|l|}{ Ring stretchings } \\
\hline $\mathrm{S}_{1}=\mathrm{r}_{1,2}$ & & $v \mathrm{~N} 1 \mathrm{C} 2$ \\
\hline $\mathrm{S}_{2}=\mathrm{r}_{2,3}$ & & $v \mathrm{C} 2 \mathrm{~N} 3$ \\
\hline $\mathrm{S}_{3}=\mathrm{r}_{3,4}$ & & $v \mathrm{~N} 3 \mathrm{C} 4$ \\
\hline $\mathrm{S}_{4}=\mathrm{r}_{4,5}$ & & $v \mathrm{C} 4 \mathrm{C} 5$ \\
\hline $\mathrm{S}_{5}=\mathrm{r}_{5,6}$ & & $v \mathrm{C} 5 \mathrm{C} 6$ \\
\hline $\mathrm{S}_{6}=\mathrm{r}_{6,1}$ & & $v \mathrm{C} 6 \mathrm{~N} 1$ \\
\hline $\mathrm{S}_{7}=\mathrm{r}_{5,7}$ & & $v \mathrm{C} 5 \mathrm{~N} 7$ \\
\hline $\mathrm{S}_{8}=\mathrm{r}_{7,8}$ & & $v \mathrm{~N} 7 \mathrm{C} 8$ \\
\hline $\mathrm{S}_{9}=\mathrm{r}_{8,9}$ & & $v \mathrm{C} 8 \mathrm{~N} 9$ \\
\hline $\mathrm{S}_{10}=\mathrm{r}_{9,4}$ & & $v \mathrm{~N} 9 \mathrm{C} 4$ \\
\hline \multicolumn{3}{|l|}{ Stretching CO } \\
\hline $\mathrm{S}_{11}=\mathrm{r}_{10,6}$ & & $v \mathrm{C}-\mathrm{O}$ \\
\hline \multicolumn{3}{|l|}{ Stretching OH, CH, NH } \\
\hline $\mathrm{S}_{12}=\mathrm{r}_{11,10}$ & & $v \mathrm{OH}$ \\
\hline $\mathrm{S}_{13}=\mathrm{r}_{12,2}$ & & $v \mathrm{C} 2 \mathrm{H}$ \\
\hline $\mathrm{S}_{14}=\mathrm{r}_{13,8}$ & & $v \mathrm{C} 8 \mathrm{H}$ \\
\hline $\mathrm{S}_{15}=\mathrm{r}_{14,9}$ & III & $v \mathrm{~N} 9 \mathrm{H}$ \\
\hline $\mathrm{S}_{15}^{\prime}=\mathrm{r}_{14,7}$ & IV & $v \mathrm{~N} 7 \mathrm{H}$ \\
\hline \multicolumn{3}{|l|}{ Ring in-plane deformation } \\
\hline$S_{16}=\left(6^{-1 / 2}\right)\left(\beta_{1,3,2}-\beta_{2,4,3}+\beta_{3,5,4}-\beta_{4,6,5}+\beta_{5,1,6}-\beta_{6,2,1}\right)$ & & $\beta \mathrm{R} 1$ \\
\hline$S_{17}=\left(12^{-1 / 2}\right)\left(2 \beta_{1,3,2}-\beta_{2,4,3}-\beta_{3,5,4}+2 \beta_{4,6,5}-\beta_{5,1,6}-\beta_{6,2,1}\right)$ & & $\beta \mathrm{R} 2$ \\
\hline$S_{18}=(1 / 2)\left(\beta_{2,4,3}-\beta_{3,5,4}+\beta_{5,1,6}-\beta_{6,2,1}\right)$ & & $\beta \mathrm{R} 3$ \\
\hline $\mathrm{S}_{19}=\left(\left(1+2 a^{2}+2 b^{2}\right)^{-1 / 2}\right)\left(\beta_{7,9,8}+a\left(\beta_{5,8,7}+\beta_{8,4,9}\right)+b\left(\beta_{4,7,5}+\beta_{9,5,4}\right)\right)$ & & $\beta \mathrm{R} 4$ \\
\hline $\mathrm{S}_{20}=\left(\left(2(a-b)^{2}+2(1-a)^{2}\right)^{-1 / 2}\right)\left((a-b)\left(\beta_{5,8,7}-\beta_{8,4,9}\right)+(1-a)\left(\beta_{4,7,5}-\beta_{9,5,4}\right)\right)$ & & $\beta \mathrm{R} 5$ \\
\hline \multicolumn{3}{|l|}{ Bendings CO, CH, NH, OH } \\
\hline$S_{21}=\left(2^{-1 / 2}\right)\left(\beta_{1,10,6}-\beta_{5,10,6}\right)$ & & $\beta \mathrm{C}-\mathrm{O}$ \\
\hline $\mathrm{S}_{22}=\beta_{11,6,10}$ & & $\beta \mathrm{OH}$ \\
\hline$S_{23}=\left(2^{-1 / 2}\right)\left(\beta_{3,12,2}-\beta_{1,12,2}\right)$ & & $\beta \mathrm{C} 2 \mathrm{H}$ \\
\hline $\mathrm{S}_{24}=\left(2^{-1 / 2}\right)\left(\beta_{7,13,8}-\beta_{9,13,8}\right)$ & & $\beta \mathrm{C} 8 \mathrm{H}$ \\
\hline$S_{25}=\left(2^{-1 / 2}\right)\left(\beta_{8,14,9}-\beta_{4,14,9}\right)$ & III & $\beta \mathrm{N} 9 \mathrm{H}$ \\
\hline$S_{25}^{\prime}=\left(2^{-1 / 2}\right)\left(\beta_{5,14,7}-\beta_{8,14,7}\right)$ & IV & $\beta \mathrm{N} 7 \mathrm{H}$ \\
\hline \multicolumn{3}{|l|}{ Out-of-plane } \\
\hline \multicolumn{3}{|l|}{ Torsion OH } \\
\hline$S_{26}=\left(2^{-1 / 2}\right)\left(\tau_{11,10,6,1}+\tau_{11,10,6,5}\right)$ & & $\tau \mathrm{OH}$ \\
\hline \multicolumn{3}{|l|}{ Wagging CO, NH, CH } \\
\hline$S_{27}=\gamma_{10,5,6,1}$ & & $\gamma \mathrm{C}-\mathrm{O}$ \\
\hline $\mathrm{S}_{28}=\gamma_{12,1,2,3}$ & & $\gamma \mathrm{C} 2 \mathrm{H}$ \\
\hline $\mathrm{S}_{29}=\gamma_{13,7,8,9}$ & & $\gamma \mathrm{C} 8 \mathrm{H}$ \\
\hline $\mathrm{S}_{30}=\gamma_{14,8,9,4}$ & III & $\gamma \mathrm{N} 9 \mathrm{H}$ \\
\hline $\mathrm{S}_{30}^{\prime}=\gamma_{14,5,7,8}$ & IV & $\gamma \mathrm{N} 7 \mathrm{H}$ \\
\hline \multicolumn{3}{|l|}{ Ring torsions } \\
\hline$S_{31}=\left(6^{-1 / 2}\right)\left(\tau_{1,2,3,4}-\tau_{2,3,4,5}+\tau_{3,4,5,6}-\tau_{4,5,6,1}+\tau_{5,6,1,2}-\tau_{6,1,2,3}\right)$ & & $\tau \mathrm{R} 1$ \\
\hline$S_{32}=\left(12^{-1 / 2}\right)\left(2 \tau_{1,2,3,4}-\tau_{2,3,4,5}-\tau_{3,4,5,6}+2 \tau_{4,5,6,1}-\tau_{5,6,1,2}-\tau_{6,1,2,3}\right)$ & & $\tau \mathrm{R} 2$ \\
\hline$S_{33}=(1 / 2)\left(\tau_{2,3,4,5}-\tau_{3,4,5,6}+\tau_{5,6,1,2}-\tau_{6,1,2,3}\right)$ & & $\tau \mathrm{R} 3$ \\
\hline $\mathrm{S}_{34}=\left(\left(1+2 a^{2}+2 b^{2}\right)^{-1 / 2}\right)\left(\tau_{4,5,7,8}+b\left(\tau_{7,8,9,4}+\tau_{8,9,4,5}\right)+a\left(\tau_{5,7,8,9}+\tau_{9,4,5,7}\right)\right)$ & & $\tau \mathrm{R} 4$ \\
\hline $\mathrm{S}_{35}=\left(\left(2(a-b)^{2}+2(1-a)^{2}\right)^{-1 / 2}\right)\left((a-b)\left(\tau_{9,4,5,7}-\tau_{5,7,8,9}\right)+(1-a)\left(\tau_{8,9,4,5}-\tau_{7,8,9,4}\right)\right)$ & & $\tau \mathrm{R} 5$ \\
\hline Two rings relative torsion & & \\
\hline$S_{36}=\left(2^{-1 / 2}\right)\left(\tau_{6,5,4,9}-\tau_{7,5,4,3}\right)$ & & $\tau \mathrm{RR}$ \\
\hline
\end{tabular}

$r_{i, j}$ is the distance between atoms $A_{i}$ and $A_{j} ; \beta_{i, j, k}$ is the angle between vectors $A_{k} A_{i}$ and $A_{k} A_{j} ; \tau_{i, j, k, l}$ is the dihedral angle between the plane defined by $A_{i}, A_{j}, A_{k}$ and the plane defined by $A_{j}, A_{k}, A_{l}$ atoms; $\gamma_{i, j, k, l}$ is the angle between the vector $A_{k} A_{i}$ and the plane defined by atoms $A_{j}, A_{k}, A_{l}$. $a=\cos 144^{\circ}=-0.8090 ; b=\cos 72^{\circ}=0.3090$ 
TABLE S3: Calculated vibrational frequencies, intensities and potential energy distributions for hypoxanthine form I (frequencies ( $\tilde{v})$ in $\mathrm{cm}^{-1}$, intensities $\left(\mathrm{A}^{\text {th }}\right)$ in $\mathrm{km} \mathrm{mol}^{-1}$, PED's in \%). Theoretical positions of absorption bands were scaled by a factor of 0.98 . PED's lower than $10 \%$ are not included. Definition of internal coordinates is given in Table S1.

\begin{tabular}{|c|c|c|c|}
\hline \multicolumn{4}{|c|}{ Normal vibrations calculated at the DFT(B3LYP)/6-31++G(d,p) level } \\
\hline $\mathbf{N r}$ & $\tilde{v}$ & $\mathbf{A}^{\text {th }}$ & PED \\
\hline Q1 & 3582 & 89 & $v \mathrm{~N} 9 \mathrm{H}(100)$ \\
\hline Q2 & 3528 & 66 & $v \mathrm{~N} 1 \mathrm{H}(100)$ \\
\hline Q3 & 3202 & 0 & $v \mathrm{C} 8 \mathrm{H}(99)$ \\
\hline Q4 & 3137 & 4 & $v \mathrm{C} 2 \mathrm{H}(100)$ \\
\hline Q5 & 1764 & 723 & $v \mathrm{C}=\mathrm{O}(73), v \mathrm{C} 5 \mathrm{C} 6(13)$ \\
\hline Q6 & 1598 & 102 & $v \mathrm{C} 2 \mathrm{~N} 3(55), \beta \mathrm{C} 2 \mathrm{H}(15)$ \\
\hline Q7 & 1564 & 66 & $v \mathrm{~N} 3 \mathrm{C} 4(25), v \mathrm{C} 4 \mathrm{C} 5(18), v \mathrm{~N} 9 \mathrm{C} 4(14), \beta \mathrm{N9H}(14), v \mathrm{~N} 7 \mathrm{C} 8(14)$ \\
\hline Q8 & 1499 & 33 & $v \mathrm{~N} 7 \mathrm{C} 8(21), v \mathrm{C} 4 \mathrm{C} 5(20), \beta \mathrm{C} 8 \mathrm{H}(12)$ \\
\hline Q9 & 1454 & 4 & $v \mathrm{~N} 7 \mathrm{C} 8(24), \beta \mathrm{N} 1 \mathrm{H}(23)$ \\
\hline Q10 & 1407 & 5 & $\beta \mathrm{N} 1 \mathrm{H}(18), v \mathrm{C} 4 \mathrm{C} 5(17), \beta \mathrm{C} 2 \mathrm{H}(17), v \mathrm{~N} 1 \mathrm{C} 2(16)$ \\
\hline Q11 & 1379 & 23 & $\beta \mathrm{N} 9 \mathrm{H}(24), \beta \mathrm{C} 2 \mathrm{H}(18), v \mathrm{C} 8 \mathrm{~N} 9$ (16) \\
\hline Q12 & 1352 & 35 & $v \mathrm{C} 5 \mathrm{~N} 7(30), \beta \mathrm{C} 2 \mathrm{H}(21), v \mathrm{C} 2 \mathrm{~N} 3(14)$ \\
\hline Q13 & 1332 & 4 & $v \mathrm{C} 5 \mathrm{C} 6(15), v \mathrm{~N} 9 \mathrm{C} 4(14), \beta \mathrm{C} 8 \mathrm{H}(14), \beta \mathrm{C} 2 \mathrm{H}(12)$ \\
\hline Q14 & 1274 & 5 & $\beta \mathrm{C} 8 \mathrm{H}(31), v \mathrm{~N} 7 \mathrm{C} 8(14), \beta \mathrm{N} 9 \mathrm{H}(10), v \mathrm{~N} 3 \mathrm{C} 4(10)$ \\
\hline Q15 & 1170 & 80 & $\beta \mathrm{C} 8 \mathrm{H}(18), \beta \mathrm{R} 1(14), v \mathrm{~N} 1 \mathrm{C} 2(11)$ \\
\hline Q16 & 1115 & 11 & $v \mathrm{~N} 1 \mathrm{C} 2(41), \beta \mathrm{N} 1 \mathrm{H}(21)$ \\
\hline Q17 & 1061 & 18 & $v$ C8N9 (53), $\beta$ N9H (31) \\
\hline Q18 & 1045 & 41 & $v \mathrm{C} 6 \mathrm{~N} 1(34), \beta \mathrm{C}=\mathrm{O}(21)$ \\
\hline Q19 & 926 & 5 & $\beta \mathrm{R} 4$ (76), $v \mathrm{C} 4 \mathrm{C} 5(11)$ \\
\hline Q20 & 914 & 3 & $\gamma \mathrm{C} 2 \mathrm{H}(107)$ \\
\hline Q21 & 884 & 9 & $\beta \mathrm{R} 1(52), \beta \mathrm{R} 2(13)$ \\
\hline Q22 & 821 & 15 & $\gamma \mathrm{C} 8 \mathrm{H}(100)$ \\
\hline Q23 & 759 & 8 & $\gamma \mathrm{C}=\mathrm{O}(34), \tau \mathrm{R} 1(32), \tau \mathrm{R} 4(27)$ \\
\hline Q24 & 713 & 46 & $\gamma \mathrm{N} 1 \mathrm{H}(48), \gamma \mathrm{C}=\mathrm{O}(38)$ \\
\hline Q25 & 688 & 10 & $v \mathrm{~N} 3 \mathrm{C} 4(22), v \mathrm{C} 6 \mathrm{~N} 1(19), v \mathrm{~N} 9 \mathrm{C} 4(12)$ \\
\hline Q26 & 656 & 18 & $\tau \mathrm{R} 5$ (56), $\tau \mathrm{R} 4(23), \gamma \mathrm{N} 1 \mathrm{H}(14)$ \\
\hline Q27 & 638 & 15 & $\tau \mathrm{R} 4(32), \gamma \mathrm{N} 1 \mathrm{H}(32), \gamma \mathrm{C}=\mathrm{O}(17), \tau \mathrm{R} 3(15)$ \\
\hline Q28 & 595 & 8 & $\beta \mathrm{R} 5$ (31), $v \mathrm{C} 5 \mathrm{C} 6(20), \beta \mathrm{C}=\mathrm{O}(12)$ \\
\hline Q29 & 557 & 121 & $\gamma \mathrm{N} 9 \mathrm{H}(53), \tau \mathrm{R} 2(19), \tau \mathrm{R} 1(17)$ \\
\hline Q30 & 526 & 11 & $\beta \mathrm{R} 2(41), \beta \mathrm{C}=\mathrm{O}(19), v \mathrm{C} 6 \mathrm{~N} 1$ (11), $\beta \mathrm{R} 1(10)$ \\
\hline Q31 & 516 & 13 & $\gamma \mathrm{N} 9 \mathrm{H}(48), \tau \mathrm{R} 2(22), \tau \mathrm{R} 5(16), \tau \mathrm{R} 1$ (12) \\
\hline Q32 & 498 & 2 & $\beta \mathrm{R} 3(69)$ \\
\hline Q33 & 315 & 1 & $\beta \mathrm{C}=\mathrm{O}(38), \beta \mathrm{R} 2(17), v \mathrm{C} 5 \mathrm{C} 6(12)$ \\
\hline Q34 & 263 & 0 & $\tau \mathrm{R} 3(58), \tau \mathrm{RR}(20), \tau \mathrm{R} 5(15)$ \\
\hline Q35 & 203 & 17 & $\tau \mathrm{RR}(63), \tau \mathrm{R} 3(28)$ \\
\hline Q36 & 149 & 1 & $\tau \mathrm{R} 2(59), \tau \mathrm{R} 1(31)$ \\
\hline
\end{tabular}


TABLE S4: Calculated vibrational frequencies, intensities and potential energy distributions for hypoxanthine form II (frequencies ( $\tilde{v})$ in $\mathrm{cm}^{-1}$, intensities $\left(\mathrm{A}^{\text {th }}\right)$ in $\mathrm{km} \mathrm{mol}^{-1}$, PED's in \%). Theoretical positions of absorption bands were scaled by a factor of 0.98 . PED's lower than $10 \%$ are not included. Definition of internal coordinates is given in Table S1.

\begin{tabular}{|c|c|c|c|}
\hline \multicolumn{4}{|c|}{ Normal vibrations calculated at the DFT(B3LYP)/6-31++G(d,p) level } \\
\hline $\mathbf{N r}$ & $\tilde{v}$ & $\mathbf{A}^{\text {th }}$ & PED \\
\hline Q1 & 3582 & 102 & $v \mathrm{~N} 7 \mathrm{H}(100)$ \\
\hline Q2 & 3531 & 70 & $v \mathrm{~N} 1 \mathrm{H}(100)$ \\
\hline Q3 & 3203 & 1 & $v \mathrm{C} 8 \mathrm{H}(99)$ \\
\hline Q4 & 3134 & 6 & $v \mathrm{C} 2 \mathrm{H}(100)$ \\
\hline Q5 & 1739 & 796 & $v \mathrm{C}=\mathrm{O}(64), v \mathrm{C} 5 \mathrm{C} 6(16)$ \\
\hline Q6 & 1608 & 75 & $v \mathrm{C} 2 \mathrm{~N} 3(52), \beta \mathrm{C} 2 \mathrm{H}(10)$ \\
\hline Q7 & 1526 & 30 & $v \mathrm{C} 4 \mathrm{C} 5(18), \beta \mathrm{N} 7 \mathrm{H}(14), v \mathrm{C} 2 \mathrm{~N} 3(12), v \mathrm{C} 8 \mathrm{~N} 9(11), v \mathrm{C} 5 \mathrm{~N} 7(10)$ \\
\hline Q8 & 1515 & 9 & $v \mathrm{C} 4 \mathrm{C} 5(27), v \mathrm{~N} 3 \mathrm{C} 4(25)$ \\
\hline Q9 & 1441 & 36 & $v \mathrm{C} 8 \mathrm{~N} 9(32), \beta \mathrm{C} 8 \mathrm{H}(15), \beta \mathrm{R} 5$ (13), $v \mathrm{C} 5 \mathrm{~N} 7$ (11) \\
\hline Q10 & 1410 & 25 & $\beta \mathrm{N} 1 \mathrm{H}(39), \beta \mathrm{N} 7 \mathrm{H}(16), v \mathrm{~N} 1 \mathrm{C} 2(16), v \mathrm{~N} 7 \mathrm{C} 8(11)$ \\
\hline Q11 & 1387 & 33 & $\beta \mathrm{C} 2 \mathrm{H}(45), v \mathrm{C} 5 \mathrm{~N} 7(11), v \mathrm{C} 2 \mathrm{~N} 3(10), v \mathrm{C} 4 \mathrm{C} 5(10), v \mathrm{~N} 3 \mathrm{C} 4(10)$ \\
\hline Q12 & 1378 & 90 & $v \mathrm{~N} 7 \mathrm{C} 8(16), v \mathrm{~N} 9 \mathrm{C} 4(13), \beta \mathrm{N} 7 \mathrm{H}(13), v \mathrm{C} 8 \mathrm{~N} 9(11)$ \\
\hline Q13 & 1325 & 28 & $\beta \mathrm{C} 2 \mathrm{H}(29), v \mathrm{~N} 9 \mathrm{C} 4(22), v \mathrm{C} 5 \mathrm{~N} 7(13), v \mathrm{~N} 3 \mathrm{C} 4(12)$ \\
\hline Q14 & 1273 & 0 & $\beta \mathrm{C} 8 \mathrm{H}(31), v \mathrm{C} 8 \mathrm{~N} 9(24), v \mathrm{C} 5 \mathrm{C} 6(11), v \mathrm{~N} 9 \mathrm{C} 4(10)$ \\
\hline Q15 & 1184 & 75 & $\beta \mathrm{C} 8 \mathrm{H}(18), \beta \mathrm{R} 1(18), v \mathrm{C} 6 \mathrm{~N} 1$ (15), $v \mathrm{C} 5 \mathrm{~N} 7$ (10) \\
\hline Q16 & 1101 & 8 & $v \mathrm{~N} 1 \mathrm{C} 2(43), \beta \mathrm{N} 1 \mathrm{H}(20)$ \\
\hline Q17 & 1080 & 43 & $v \mathrm{~N} 7 \mathrm{C} 8(48), \beta \mathrm{N} 7 \mathrm{H}(23), \beta \mathrm{C} 8 \mathrm{H}(10)$ \\
\hline Q18 & 1060 & 14 & $v \mathrm{C} 6 \mathrm{~N} 1(21), \beta \mathrm{R} 5(14), \beta \mathrm{C}=\mathrm{O}(13)$ \\
\hline Q19 & 938 & 1 & $\beta \mathrm{R} 4(73), v \mathrm{C} 4 \mathrm{C} 5(13)$ \\
\hline Q20 & 910 & 6 & $\gamma \mathrm{C} 2 \mathrm{H}(107)$ \\
\hline Q21 & 882 & 3 & $\beta \mathrm{R} 1(53), \beta \mathrm{R} 2(15)$ \\
\hline Q22 & 845 & 13 & $\gamma \mathrm{C} 8 \mathrm{H}(103)$ \\
\hline Q23 & 757 & 5 & $\tau \mathrm{R} 1(43), \tau \mathrm{R} 5(27), \tau \mathrm{R} 4(13), \gamma \mathrm{C}=\mathrm{O}(12)$ \\
\hline Q24 & 716 & 32 & $\gamma \mathrm{C}=\mathrm{O}(69), \gamma \mathrm{N} 1 \mathrm{H}(25), \tau \mathrm{R} 5(10)$ \\
\hline Q25 & 697 & 3 & $v \mathrm{~N} 3 \mathrm{C} 4(25), v \mathrm{C} 6 \mathrm{~N} 1(16), v \mathrm{~N} 9 \mathrm{C} 4(10)$ \\
\hline Q26 & 658 & 28 & $\gamma \mathrm{N} 1 \mathrm{H}(46), \tau \mathrm{R} 5(32), \tau \mathrm{R} 4(13)$ \\
\hline Q27 & 620 & 41 & $\tau \mathrm{R} 4(41), \gamma \mathrm{N} 1 \mathrm{H}(21), \gamma \mathrm{N} 7 \mathrm{H}(12), \tau \mathrm{R} 3(11), \gamma \mathrm{C}=\mathrm{O}(11)$ \\
\hline Q28 & 600 & 6 & $\beta \mathrm{R} 5(33), v \mathrm{C} 5 \mathrm{C} 6(17), \beta \mathrm{C}=\mathrm{O}(13)$ \\
\hline Q29 & 547 & 20 & $\tau \mathrm{R} 2(39), \tau \mathrm{R} 1$ (29), $\tau \mathrm{R} 4(12), \tau \mathrm{R} 5(12), \gamma \mathrm{N} 1 \mathrm{H}(11)$ \\
\hline Q30 & 538 & 3 & $\beta \mathrm{R} 2(35), \beta \mathrm{C}=\mathrm{O}(22), v \mathrm{C} 6 \mathrm{~N} 1$ \\
\hline Q31 & 516 & 84 & $\gamma \mathrm{N} 7 \mathrm{H}(86)$ \\
\hline Q32 & 504 & 3 & $\beta \mathrm{R} 3(68), \beta \mathrm{R} 2(11)$ \\
\hline Q33 & 300 & 17 & $\beta \mathrm{C}=\mathrm{O}(39), \beta \mathrm{R} 2(17), v \mathrm{C} 5 \mathrm{C} 6(12)$ \\
\hline Q34 & 270 & 5 & $\tau \mathrm{R} 3$ (54), $\tau \mathrm{RR}(25), \tau \mathrm{R} 4(12)$ \\
\hline Q35 & 191 & 12 & $\tau \mathrm{RR}(54), \tau \mathrm{R} 3(37), \tau \mathrm{R} 2(10)$ \\
\hline Q36 & 156 & 15 & $\tau \mathrm{R} 2(52), \tau \mathrm{R} 1(36)$ \\
\hline
\end{tabular}


TABLE S5: Calculated vibrational frequencies, intensities and potential energy distributions for hypoxanthine form III (frequencies ( $(\tilde{v})$ in $\mathrm{cm}^{-1}$, intensities $\left(\mathrm{A}^{\text {th }}\right)$ in $\mathrm{km} \mathrm{mol}^{-1}$, PED's in \%). Theoretical positions of absorption bands were scaled by a factor of 0.98 . PED's lower than $10 \%$ are not included. Definition of internal coordinates is given in Table S2.

\begin{tabular}{|c|c|c|c|}
\hline \multicolumn{4}{|c|}{ Normal vibrations calculated at the DFT(B3LYP)/6-31++G(d,p) level } \\
\hline $\mathbf{N r}$ & $\tilde{v}$ & $\mathrm{~A}^{\mathrm{th}}$ & PED \\
\hline Q1 & 3681 & 104 & $v \mathrm{OH}(100)$ \\
\hline Q2 & 3587 & 94 & $v \mathrm{~N} 9 \mathrm{H}(100)$ \\
\hline Q3 & 3198 & 0 & $v \mathrm{C} 8 \mathrm{H}(99)$ \\
\hline Q4 & 3138 & 13 & $v \mathrm{C} 2 \mathrm{H}(100)$ \\
\hline Q5 & 1638 & 168 & $v \mathrm{C} 5 \mathrm{C} 6(35), v \mathrm{~N} 3 \mathrm{C} 4(11)$ \\
\hline Q6 & 1597 & 238 & $v \mathrm{C} 4 \mathrm{C} 5(23), v \mathrm{~N} 3 \mathrm{C} 4(20), v \mathrm{~N} 1 \mathrm{C} 2(11)$ \\
\hline Q7 & 1499 & 26 & $v \mathrm{~N} 7 \mathrm{C} 8(49), \beta \mathrm{C} 8 \mathrm{H}(21)$ \\
\hline Q8 & 1477 & 158 & $v \mathrm{C} 6 \mathrm{~N} 1(29), \beta \mathrm{C} 2 \mathrm{H}(26), v \mathrm{C}-\mathrm{O}(14), v \mathrm{C} 2 \mathrm{~N} 3(13)$ \\
\hline Q9 & 1415 & 29 & $v \mathrm{~N} 9 \mathrm{C} 4(28), v \mathrm{C} 4 \mathrm{C} 5(20), v \mathrm{~N} 7 \mathrm{C} 8(11)$ \\
\hline Q10 & 1393 & 8 & $\beta \mathrm{C} 2 \mathrm{H}(33), \beta \mathrm{N} 9 \mathrm{H}(31), v \mathrm{C} 8 \mathrm{~N} 9$ (17) \\
\hline Q11 & 1340 & 90 & $v \mathrm{C} 4 \mathrm{C} 5(16), \beta \mathrm{C} 8 \mathrm{H}(16), v \mathrm{C} 6 \mathrm{~N} 1(11), v \mathrm{C} 5 \mathrm{~N} 7(10)$ \\
\hline Q12 & 1335 & 150 & $v \mathrm{C}-\mathrm{O}(17), v \mathrm{C} 5 \mathrm{~N} 7(15), v \mathrm{C} 2 \mathrm{~N} 3(15), \beta \mathrm{R} 1(14)$ \\
\hline Q13 & 1311 & 7 & $v \mathrm{C} 2 \mathrm{~N} 3(35), v \mathrm{~N} 1 \mathrm{C} 2(28), \beta \mathrm{C} 2 \mathrm{H}(17)$ \\
\hline Q14 & 1275 & 23 & $\beta \mathrm{OH}(42), v \mathrm{C} 5 \mathrm{~N} 7(25)$ \\
\hline Q15 & 1249 & 23 & $\beta \mathrm{C} 8 \mathrm{H}(38), v \mathrm{~N} 7 \mathrm{C} 8(17), \beta \mathrm{N} 9 \mathrm{H}(10)$ \\
\hline Q16 & 1131 & 2 & $\beta \mathrm{R} 5(14), v \mathrm{~N} 1 \mathrm{C} 2(13), v \mathrm{C} 2 \mathrm{~N} 3(10)$ \\
\hline Q17 & 1081 & 185 & $v \mathrm{C} 6 \mathrm{~N} 1(21), \beta \mathrm{OH}(18), v \mathrm{C}-\mathrm{O}(13), \beta \mathrm{R} 5(10)$ \\
\hline Q18 & 1061 & 11 & $\nu \mathrm{C} 8 \mathrm{~N} 9(56), \beta \mathrm{N} 9 \mathrm{H}(32)$ \\
\hline Q19 & 948 & 5 & $\gamma \mathrm{C} 2 \mathrm{H}(108)$ \\
\hline Q20 & 921 & 14 & $\beta \mathrm{R} 4(73), v \mathrm{C} 4 \mathrm{C} 5(13)$ \\
\hline Q21 & 880 & 15 & $\beta \mathrm{R} 1(49), \beta \mathrm{R} 2(20)$ \\
\hline Q22 & 847 & 10 & $\gamma \mathrm{C} 8 \mathrm{H}(100)$ \\
\hline Q23 & 785 & 11 & $\tau \mathrm{R} 1$ (52), $\tau \mathrm{R} 4$ (22), $\gamma \mathrm{C}-\mathrm{O}(18)$ \\
\hline Q24 & 712 & 2 & $v \mathrm{~N} 3 \mathrm{C} 4(19), v \mathrm{~N} 9 \mathrm{C} 4(11)$ \\
\hline Q25 & 676 & 13 & $\gamma \mathrm{C}-\mathrm{O}(49), \tau \mathrm{R} 3(14), \tau \mathrm{R} 5(13), \tau \mathrm{R} 4(11), \tau \mathrm{RR}(10)$ \\
\hline Q26 & 652 & 11 & $\tau \mathrm{R} 4(48), \tau \mathrm{R} 5(40)$ \\
\hline Q27 & 607 & 1 & $\beta \mathrm{R} 5$ (36), $v \mathrm{C} 5 \mathrm{C} 6(19), \beta \mathrm{R} 2(10)$ \\
\hline Q28 & 567 & 37 & $\tau \mathrm{R} 2(29), \tau \mathrm{R} 1(23), \tau \mathrm{R} 5$ (18), $\gamma \mathrm{N} 9 \mathrm{H}(16)$ \\
\hline Q29 & 545 & 135 & $\tau \mathrm{OH}(88)$ \\
\hline Q30 & 525 & 8 & $\beta \mathrm{C}-\mathrm{O}(28), \beta \mathrm{R} 2(22), v \mathrm{~N} 9 \mathrm{C} 4(13), \beta \mathrm{R} 3(11)$ \\
\hline Q31 & 520 & 0 & $\beta \mathrm{R} 3(54), \beta \mathrm{R} 2(21)$ \\
\hline Q32 & 508 & 40 & $\gamma \mathrm{N9H}(79)$ \\
\hline Q33 & 297 & 13 & $\beta \mathrm{C}-\mathrm{O}(48), \beta \mathrm{R} 2(12)$ \\
\hline Q34 & 296 & 0 & $\tau \mathrm{R} 3(59), \tau \mathrm{R} 5(16)$ \\
\hline Q35 & 216 & 12 & $\tau \mathrm{RR}(70), \tau \mathrm{R} 3(17)$ \\
\hline Q36 & 162 & 3 & $\tau \mathrm{R} 2(64), \tau \mathrm{R} 1(17), \gamma \mathrm{C}-\mathrm{O}(12)$ \\
\hline
\end{tabular}


TABLE S6: Calculated vibrational frequencies, intensities and potential energy distributions for hypoxanthine form IV (frequencies $(\tilde{\mathrm{v}})$ in $\mathrm{cm}^{-1}$, intensities $\left(\mathrm{A}^{\text {th }}\right)$ in $\mathrm{km} \mathrm{mol}^{-1}$, PED's in \%). Theoretical positions of absorption bands were scaled by a factor of 0.98 . PED's lower than $10 \%$ are not included. Definition of internal coordinates is given in Table S2.

\begin{tabular}{|c|c|c|c|}
\hline \multicolumn{4}{|c|}{ Normal vibrations calculated at the DFT(B3LYP)/6-31++G(d, p) level } \\
\hline $\mathbf{N r}$ & $\tilde{v}$ & $\mathrm{~A}^{\mathrm{th}}$ & PED \\
\hline Q1 & 3683 & 104 & $v \mathrm{OH}(100)$ \\
\hline Q2 & 3593 & 95 & $v \mathrm{~N} 7 \mathrm{H}(100)$ \\
\hline Q3 & 3196 & 1 & $v \mathrm{C} 8 \mathrm{H}(99)$ \\
\hline Q4 & 3137 & 15 & $v \mathrm{C} 2 \mathrm{H}(100)$ \\
\hline Q5 & 1662 & 167 & $v \mathrm{C} 5 \mathrm{C} 6(43), v \mathrm{C} 5 \mathrm{~N} 7(13)$ \\
\hline Q6 & 1566 & 260 & $v \mathrm{~N} 3 \mathrm{C} 4(24), v \mathrm{C} 6 \mathrm{~N} 1(23), v \mathrm{C} 4 \mathrm{C} 5(22)$ \\
\hline Q7 & 1504 & 50 & $\nu \mathrm{C} 8 \mathrm{~N} 9(52), \beta \mathrm{C} 8 \mathrm{H}(19)$ \\
\hline Q8 & 1485 & 24 & $\beta \mathrm{C} 2 \mathrm{H}(35), v \mathrm{C} 2 \mathrm{~N} 3(17), v \mathrm{C} 6 \mathrm{~N} 1(15)$ \\
\hline Q9 & 1399 & 136 & $\beta \mathrm{N} 7 \mathrm{H}(40), v \mathrm{~N} 7 \mathrm{C} 8(24)$ \\
\hline Q10 & 1380 & 128 & $v \mathrm{C} 4 \mathrm{C} 5(20), v \mathrm{C} 2 \mathrm{~N} 3(19), v \mathrm{C} 5 \mathrm{~N} 7(18), v \mathrm{~N} 3 \mathrm{C} 4(13), \beta \mathrm{C} 2 \mathrm{H}(10)$ \\
\hline Q11 & 1362 & 53 & $v \mathrm{~N} 9 \mathrm{C} 4(18), v \mathrm{C} 4 \mathrm{C} 5(13), \beta \mathrm{R} 1(11), v \mathrm{C} 2 \mathrm{~N} 3(11), v \mathrm{C} 8 \mathrm{~N} 9(10)$ \\
\hline Q12 & 1337 & 47 & $\beta \mathrm{C} 2 \mathrm{H}(45), v \mathrm{~N} 9 \mathrm{C} 4(13), \beta \mathrm{OH}(11), v \mathrm{~N} 3 \mathrm{C} 4(10)$ \\
\hline Q13 & 1303 & 5 & $v \mathrm{C} 2 \mathrm{~N} 3(29), v \mathrm{C} 5 \mathrm{~N} 7(14), \beta \mathrm{OH}(13), v \mathrm{C} 8 \mathrm{~N} 9(12)$ \\
\hline Q14 & 1266 & 22 & $v \mathrm{~N} 1 \mathrm{C} 2(40), \beta \mathrm{OH}(13)$ \\
\hline Q15 & 1239 & 49 & $\beta \mathrm{C} 8 \mathrm{H}(35), \beta \mathrm{OH}(15), v \mathrm{C} 8 \mathrm{~N} 9(13), \beta \mathrm{N} 7 \mathrm{H}(10)$ \\
\hline Q16 & 1130 & 21 & $v \mathrm{~N} 1 \mathrm{C} 2(20), \beta \mathrm{OH}(16), \beta \mathrm{R} 5(11)$ \\
\hline Q17 & 1084 & 46 & $v \mathrm{~N} 7 \mathrm{C} 8(48), \beta \mathrm{N} 7 \mathrm{H}(17)$ \\
\hline Q18 & 1055 & 146 & $v \mathrm{C}-\mathrm{O}(15), \beta \mathrm{R} 5(14), \beta \mathrm{N} 7 \mathrm{H}(13)$ \\
\hline Q19 & 949 & 6 & $\gamma \mathrm{C} 2 \mathrm{H}(108)$ \\
\hline Q20 & 929 & 3 & $\beta \mathrm{R} 4(70), v \mathrm{C} 4 \mathrm{C} 5(17)$ \\
\hline Q21 & 872 & 20 & $\beta \mathrm{R} 1$ (47), $\beta$ R2 (19) \\
\hline Q22 & 865 & 14 & $\gamma \mathrm{C} 8 \mathrm{H}(103)$ \\
\hline Q23 & 781 & 12 & $\tau \mathrm{R} 1(56), \tau \mathrm{R} 5$ (21), $\tau \mathrm{R} 4(11)$ \\
\hline Q24 & 713 & 2 & $v \mathrm{~N} 3 \mathrm{C} 4(22), v \mathrm{~N} 9 \mathrm{C} 4(11), \beta \mathrm{R} 5(10)$ \\
\hline Q25 & 684 & 5 & $\gamma \mathrm{C}-\mathrm{O}(48), \tau \mathrm{R} 5(31), \tau \mathrm{RR}(12)$ \\
\hline Q26 & 623 & 3 & $\tau \mathrm{R} 4(58), \tau \mathrm{R} 5(17)$ \\
\hline Q27 & 606 & 1 & $\beta \mathrm{R} 5$ (36), $v$ C5C6 (19) \\
\hline Q28 & 572 & 0 & $\tau \mathrm{R} 2(35), \tau \mathrm{R} 1(25), \gamma \mathrm{C}-\mathrm{O}(15), \tau \mathrm{R} 5$ \\
\hline Q29 & 536 & 0 & $\beta \mathrm{C}-\mathrm{O}(26), \beta \mathrm{R} 3(23), \beta \mathrm{R} 2(16), v \mathrm{~N} 9 \mathrm{C} 4(15)$ \\
\hline Q30 & 518 & 1 & $\beta$ R3 (42), $\beta$ R2 (30) \\
\hline Q31 & 512 & 138 & $\tau \mathrm{OH}(93)$ \\
\hline Q32 & 449 & 83 & $\gamma \mathrm{N} 7 \mathrm{H}(93)$ \\
\hline Q33 & 299 & 4 & $\tau \mathrm{R} 3(54), \tau \mathrm{R} 4(15), \tau \mathrm{RR}(14)$ \\
\hline Q34 & 287 & 13 & $\beta \mathrm{C}-\mathrm{O}(49), \beta \mathrm{R} 2(11)$ \\
\hline Q35 & 212 & 7 & $\tau \mathrm{RR}(62), \tau \mathrm{R} 3(19), \tau \mathrm{R} 2(15)$ \\
\hline Q36 & 165 & 4 & $\tau \mathrm{R} 2(57), \tau \mathrm{R} 1(20), \gamma \mathrm{C}-\mathrm{O}(12)$ \\
\hline
\end{tabular}


TABLE S7: Spectral positions $\left(\mathrm{cm}^{-1}\right)$ of IR bands due to unidentified photoproducts generated upon UV-irradiation of hypoxanthine isolated in an argon matrix.

\begin{tabular}{|l|l|l|l|l|l|l|l|}
\hline 2144 & 1673 & 1590 & 1476 & 1355 & 1137 & 822 & 718 \\
\end{tabular}

